\title{
Ansiedad por la Imagen Corporal en Universitarios de Ciencias de la Salud Comparaciones por Sexo
}

\author{
M.C. Guadalupe Simanga Ivett Robles Hernández, \\ Dra. Susana Ivonne Aguirre Vásquez, \\ M.C. María de Jesús Muñoz Daw, \\ M.C. Edgar Francisco Ordoñez Bencomo, \\ Dr. José René Blanco Ornelas, \\ M.P.P. Jesús Omar Salido Angulo, \\ M.C. Juan de Dios Alvarado Prieto, \\ Dra. Perla Jannet Jurado García, \\ Facultad de Ciencias de la Cultura Física \\ Universidad Autónoma de Chihuahua, México
}

Doi:10.19044/esj.2020.v16n29p42 㞷L:http://dx.doi.org/10.19044/esj.2020.v16n29p42

\section{Resumen}

El objetivo de esta investigación consistió comparar los perfiles de ansiedad por la imagen corporal de mujeres y hombres universitarios de ciencias de la salud. La muestra total fue de 443 universitarios; 201 mujeres y 242 hombres, con edades entre los 17 y 20 años. El abordaje adoptado en la investigación se enmarcó dentro de un enfoque cuantitativo con un diseño descriptivo tipo encuesta. Los resultados del análisis multivariante de la varianza, seguido por los análisis de varianza univariados, muestran que en general las mujeres manifiestan una mayor ansiedad en relación a las partes de su cuerpo que tienen que ver con peso corporal $(\mathrm{F}=20.87, \mathrm{p}<.001)$ mientras que los hombres lo hacen por partes de su aspecto físico que no guardan relación con su peso corporal $(\mathrm{F}=17.74, \mathrm{p}<.001)$. No obstante, es preciso desarrollar más investigación al respecto pues el tema trasciende del todo los alcances de la presente investigación.

Palabras clave: Imagen corporal, sexo, actitud, autopercepcione 


\title{
Body Image Anxiety in Health Sciences University Students: Comparisons by Sex
}

\author{
M.C. Guadalupe Simanga Ivett Robles Hernández, \\ Dra. Susana Ivonne Aguirre Vásquez, \\ M.C. María de Jesús Muñoz Daw, \\ M.C. Edgar Francisco Ordoñez Bencomo, \\ Dr. José René Blanco Ornelas, \\ M.P.P. Jesús Omar Salido Angulo, \\ M.C. Juan de Dios Alvarado Prieto, \\ Dra. Perla Jannet Jurado García, \\ Facultad de Ciencias de la Cultura Física \\ Universidad Autónoma de Chihuahua, México
}

\begin{abstract}
This paper focuses on comparing the anxiety profiles of body image in women and men of health sciences university students. A total sample of 443 university students (201 women and 242 men) aged between 17 to 20 years was used in this study. The approach adopted in the research was framed within a quantitative approach with a descriptive survey-type design. The results of the multivariate analysis of variance, followed by the univariate analyzes of variance, show that in general women manifest greater anxiety in relation to the parts of their body that have to do with body weight $(\mathrm{F}=20.87$, $\mathrm{p}<.001$ ), while men do so for parts of their physical appearance that are not related to their body weight $(\mathrm{F}=17.74, \mathrm{p}<.001)$. It is, therefore, important to carry out more research in this regard since the subject completely transcends the scope of the present study.
\end{abstract}

Keywords: Body image, sex, attitude, self-perceptions

\section{Introducción}

La imagen corporal se ha estudiado por diversos autores entre ellos se encuentra Schilder (1935), el cual, la describe como una mentalidad o ideología del cuerpo propio, es decir, la forma cómo se percibe el cuerpo de sí mismo, posteriormente está la concepción de Cash (1990) relacionando percepción, cognición, subjetividad, autoafirmaciones de agrado o desagrado con emociones generadas. Por otra parte, Rosen (1995) afirma que lo anterior 
genera acción y reacción hacia el propio cuerpo. Es decir, la imagen corporal es la imagen mentalizada e idealizada de la silueta, relacionada o no con la realidad e influye la forma de la interacción con la sociedad (Borda-Pérez et al., 2016), por los valores, creencias y actitudes relacionadas que se pueden generar de ésta (De la Serna, 2004).

En cuanto a la ansiedad, es un conjunto de manifestaciones psíquicas y físicas no relacionadas con peligros reales, es decir, se presenta por subjetividad; se puede manifestar como crisis o ser perdurable y persistente, en ocasiones sin conocer su causa. Se puede relacionar o confundirse con el miedo por el tipo de pensamientos, sensaciones y hasta reacciones fisiológicas similares (Campbell, 1986). Por lo tanto, si la ansiedad perdura puede relacionarse con conductas o rasgos de tipo obsesivos (Sierra, Ortega \& Zubeidat, 2003).

La conducta y rasgos se generan en aspectos de la personalidad, son influenciados por la autoestima y el autoconcepto principalmente, la imagen corporal se puede ver influenciada por éstos debido a que es la percepción de las dimensiones corporales que relacionan tanto lo cognitivo-afectivo como lo axiológico (Baile \& Osorio, 2008); esto puede generar las sensaciones de agrado o desagrado y las conductas de aprobación o evitación del propio cuerpo (Thompson, Heinberg, Altabe \& Tantleff-Dunn, 2002); por ello Ahrberg, Trojca, Nasrawi y Vocks (2011) lo establecen como un concepto multidimensional.

La imagen corporal se va creando de manera evolutiva, a través de la interiorización de normas de apariencia física, dependiendo la cultura, reglas, valores y cambios físicos (Salaberria, Rodríguez \& Cruz, 2007); al discrepar entre las dimensiones reales y los juicios de valor de la belleza se manifiesta una ansiedad al no cumplirlos (Sepúlveda, Gandarillas \& Carrobes, 2004). Existen estándares de belleza donde la delgadez es lo primordial, éstos son interiorizados por jóvenes, en especial mujeres, y pueden estar relacionados con alteraciones de la imagen corporal, debido a constantes preocupaciones, lo cual puede originar trastornos de la conducta alimentaria, y el ejercicio físico excesivo puede ser una conducta que dé cuenta de alguna alteración psicológica (Vaquero-Cristóbal, Alacid, Muyor \& López-Miñarro, 2013)

Así mismo, Medina-Gómez, Martínez-Martín, Escolar-Llamazares, González-Alonso y Mercado-Val (2019) en su estudio a nivel universitario, encontraron que tanto la ansiedad como la insatisfacción corporal se encuentran presentes como factores de riesgo para los trastornos de conducta alimentaria, además, que el ambiente universitario puede generar tensión emocional, en quienes presentan mayor ansiedad; donde a mayor ansiedad, mayor insatisfacción sobre la imagen corporal. En mujeres es más frecuente la situación antes mencionada, por las presiones de los estándares sociales, llegando a percibir su imagen corporal con mayor distorsión que los hombres 
(Clode \& Fuller-Tyszkiewicz, 2016); sin embargo, Bautista-Díaz, GalvánJuárez, Martínez-Fernández, Álvarez-Ríos y García-Cruz, (2017) afirman que los medios de comunicación, las culturas, los estereotipos y las creencias son elementos importantes en la construcción del ideal del cuerpo masculino, lo que puede llevar a desarrollar trastornos de la alimentación o dismorfia muscular. Cabe mencionar que al término de la universidad se presenta ansiedad respecto a la imagen corporal, así como una preocupación hacia el futuro y las exigencias que conlleva, por la búsqueda de un empleo y la imagen a presentar ante la sociedad (Shamsuddin et al., 2013). En relación al género, las mujeres son quienes presentan niveles más altos de insatisfacción con la imagen corporal que los hombres (Robles et al., 2020).

En este estudio el objetivo fue determinar las diferencias y similitudes entre hombres y mujeres universitarios mexicanos de ciencias de la salud en cuanto a sus niveles de ansiedad por la propia imagen corporal. En consecuencia, esta investigación pretende, como investigación aplicada, aportar información que se traduzca en una práctica de mayor calidad en el contexto de la atención a este tipo de problemática.

\section{Método}

\section{Participantes}

La muestra quedó constituida por 443 universitarios de las carreras de medicina, odontología y educación física que se ofrecen en la Universidad Autónoma de Chihuahua (Tabla 1), 201 mujeres y 242 hombres. La edad de los universitarios fluctuó entre los 17 y 20 años, con $\mathrm{M}=18.13 \pm 0.72$ años para las mujeres y $M=18.24 \pm 0.71$ años para los hombres. La muestra se obtuvo mediante un muestreo por conveniencia.

Tabla 1. Distribución de los participantes de acuerdo a la carrera y sexo

\begin{tabular}{lcc}
\hline & \multicolumn{2}{c}{ Sexo } \\
\cline { 2 - 3 } \multicolumn{1}{c}{ Carrera } & $\begin{array}{c}\text { Mujeres } \\
(n=201)\end{array}$ & $\begin{array}{c}\text { Hombres } \\
(n=242)\end{array}$ \\
\hline Educación Física & 80 & 140 \\
Medicina & 64 & 75 \\
Odontología & 57 & 27 \\
\hline
\end{tabular}

\section{Instrumento}

Physical Appearance State and Trait Anxiety Scale (PASTAS-M, Escala de ansiedad de rasgo y estado de apariencia física) de Reed, Thompson, Brannick y Sacco (1991), adaptado por Ornelas, Gastélum, Blanco y Peinado (2014), para población mexicana, es una encuesta tipo Likert asistida por computadora de 12 ítems que evalúa ansiedad-rasgo relacionada con el peso (6 ítems) y con zonas corporales no relacionadas con el peso (6 ítems), donde el encuestado responde, en una escala de 0 a 10, que tan 
ansioso(a), tenso(a) o nervioso(a) se siente con respecto a diferentes partes de su cuerpo (Figura 1).

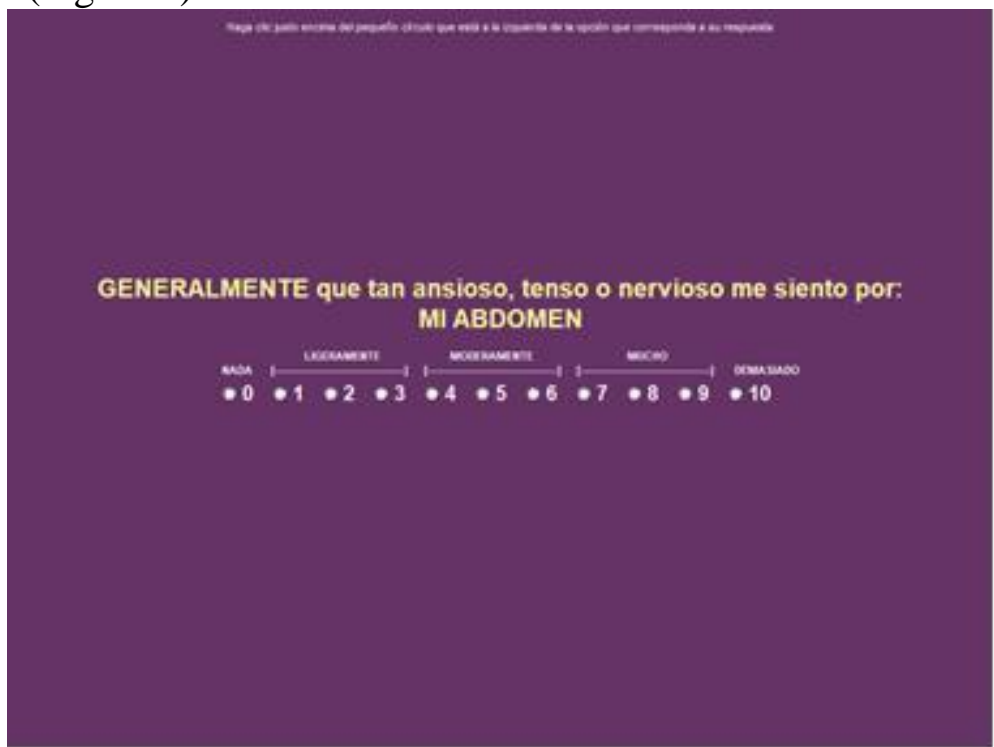

Figura 1. Ejemplo de respuesta para los ítems del cuestionario.

\section{Diseño}

En cuanto al diseño del estudio, se utilizó un enfoque cuantitativo con un diseño descriptivo y transversal tipo encuesta (Hernández, Fernández y Baptista, 2014). La variable explicativa fue el sexo y las variables respuesta el promedio de las puntuaciones obtenidas en los dos factores del cuestionario PASTAS-M (Physical Appearance State and Trait Anxiety Scale) (Factor Peso y Factor No Peso).

\section{Procedimiento}

Se invitó a participar en el estudio a alumnos de las carreras de medicina, odontología y educación física que se ofrecen en la Universidad Autónoma de Chihuahua. Los que aceptaron participar firmaron la carta de aceptación correspondiente. Luego se aplicó el instrumento, antes descrito, por medio de una computadora personal, en una sesión de aproximadamente 30 minutos. Al inicio de cada sesión se hizo una pequeña introducción sobre la importancia de la investigación y de cómo acceder al instrumento. Se les solicitó la máxima sinceridad y se les garantizó la confidencialidad de los datos que se obtuvieran. Las instrucciones de cómo responder se encontraban en las primeras pantallas; antes del primer reactivo del instrumento. Al término de la sesión se les agradeció su participación. Una vez aplicado el instrumento se procedió a recopilar los resultados por medio del módulo generador de resultados del editor de escalas versión 2.0 (Blanco et al., 2013). 


\section{Análisis de datos}

Se realizaron análisis estadísticos descriptivos (medias y desviaciones estándar) para todas las variables respuesta. Después de verificar que los datos cumplían con los supuestos de los análisis estadísticos paramétricos, Levene, Kolmogorov-Smirnov y Shapiro-Wilk, se llevó a cabo un análisis multivariante de varianza (MANOVA), seguido por los análisis de varianza univariados (ANOVAs), para examinar las diferencias entre mujeres y hombres en cuanto a su percepción de ansiedad por su imagen corporal en los factores Peso y No Peso, el tamaño del efecto se estimó mediante el eta cuadrado $\left(\eta^{2}\right)$. Todos los análisis estadísticos se realizaron con el programa SPSS versión 18.0 para Windows. El nivel de significación estadística se estableció en $p=.05$.

\section{Resultados}

La Tabla 2 muestra los valores de las medias y las desviaciones estándar de la percepción de ansiedad por la imagen corporal en los dos factores del cuestionario PASTAS-M, así como los resultados del MANOVA y los subsiguientes ANOVAs. Los resultados del MANOVA indicaron diferencias significativas globales de acuerdo a la variable sexo en las puntuaciones de percepción de ansiedad (Wilks' $\lambda=.827 ; p<.001 ; \eta^{2}=.17$ ). Posteriormente, los resultados de los ANOVAs mostraron que las mujeres reportaron una mayor percepción de ansiedad que los hombres en el Factor Peso $(F=20.87, p<.001)$, y éstos en el Factor No Peso $(F=17.74, p<.001)$.

Tabla 2. Resultados MANOVA y ANOVAs para las diferencias en percepción de ansiedad por la imagen corporal de acuerdo al sexo.

\begin{tabular}{lccccc}
\hline & $\begin{array}{c}\text { Mujeres } \\
(n=201)\end{array}$ & $\begin{array}{c}\text { Hombres } \\
(n=242)\end{array}$ & $\mathrm{F}$ & $\mathrm{p}$ & $\eta^{2}$ \\
\hline & & & 46.01 & $<.001$ & .17 \\
Factor Peso & $4.29(2.48)$ & $3.23(2.37)$ & 20.87 & $<.001$ & .05 \\
Factor No Peso & $1.01(1.45)$ & $1.71(1.96)$ & 17.74 & $<.001$ & .04 \\
\hline
\end{tabular}

Nota. Los valores descriptivos se presentan como media (desviación estándar)

\section{Discusión y Conclusiones}

Los resultados muestran que las mujeres son quienes reportan mayores niveles de ansiedad por su imagen corporal en lo que se refiere a aspectos relacionados con su peso (piernas, muslos, caderas, nalgas, abdomen y cintura), mientras que los hombres lo hacen en aspectos de su imagen corporal que no se relacionan con su peso corporal (labios, frente, manos, cuello, mentón y muñecas). Lo que concuerda con lo reportado en otras investigaciones donde se afirma que las mujeres, en comparación con hombres de la misma edad, se muestran más ansiosas en cuanto a su peso (Blanco, Aguirre, Rodríguez-Villalobos, Blanco \& Medina, 2014; Zueck, Blanco, Ornelas \& Gastélum, 2015), mientras que los hombres lo hacen por partes de 
su cuerpo que no tienen que ver por su peso (Blanco et al., 2014; Zueck et al., 2015).

Las diferencias encontradas entre hombres y mujeres con respecto a la ansiedad por su imagen corporal sugieren además, que al diseñar cualquier tipo de intervención que tenga como objetivo la disminución de la misma habrá que tomar en cuenta a la variable sexo; no obstante, es preciso desarrollar más investigación al respecto pues el tema trasciende del todo los alcances de la presente investigación. Se subraya además la importancia de realizar un mayor número de investigaciones sobre el tema en nuestro país.

Por último, cuando menos dos limitaciones están presentes en este trabajo. La primera es que los participantes son solo estudiantes universitarios chihuahuenses de ciencias de la salud, lo que supone una amenaza para la posibilidad de generalizar estos resultados. Ampliar la muestra es un área de trabajo de cara al futuro. La segunda limitación proviene del propio instrumento de medición, que se basa en el autoinforme y que por ello puede contener los sesgos que se derivan de la deseabilidad social.

\section{References:}

1. Ahrberg, M., Trojca, D., Nasrawi, N. \& Vocks, S. (2011). Body image disturbance in binge eating disorder: A review. European Eating Disorders Review, 19(5), 375-381. doi: http://doi.org/10.1002/erv.1100

2. Baile, J. I. \& Osorio, G. (2008). Relación entre la autoestima, imagen corporal y tendencia a sufrir trastorno de conducta alimentario en un grupo de adolescentes mexicanos. Revista de Psicología General Aplicada, 61(4), 397-404.

3. Bautista-Díaz, M. L., Galván-Juárez, A. K., Martínez-Fernández, I. E., Álvarez-Ríos, A. J. \& García-Cruz, R. (2017). Ideal Corporal Masculino: Percepciones De Estudiantes Universitarios De Nutrición. European Scientific Journal, 13(26), 1-19. doi: https://doi.org/10.19044/esj.2017.v13n26p1

4. Blanco, H., Aguirre, J. F., Rodríguez-Villalobos, J. M., Blanco, J. R. \& Medina, H. L. (2014). Body Image Anxiety on University Students (Differences between Men and Women). Open Journal of Medical Psychology, 3, 348-354.

5. Blanco, H., Ornelas, M., Tristán, J. L., Cocca, A., Mayorga-Vega, D., López-Walle, J. \& Viciana, J. (2013). Editor for creating and applying computerise surveys. Procedia Social and Behavioral Sciences, 106, 935-940. doi: http://dx.doi.org/10.1016/j.sbspro.2013.12.105

6. Borda-Pérez, M., Alonso-Santos, M., Martínez-Granados, H., MeriñoDía, E., Sánchez-Álvarez, J. \& Solano-Guerrero, S. (2016). Percepción de la imagen corporal y su relación con el estado nutricional y 
emocional en escolares de 10 a 13 años de tres escuelas en Barranquilla (Colombia). Revista Salud Uninorte, 32(3), 472-482. doi: http://dx.doi.org/10.14482/sun.32.3.9747

7. Campbell, S. B. (1986). Developmental issues in childhood anxiety. En R. Gittelman (Ed.), Anxiety disorders of childhood (pp. 24-57). New York: Guilford Press.

8. Cash, T. F. (1990). Body image enhancement: A program for overcoming a negative body image. New York: Guilford: Guilford.

9. Clode, J., Lewis, V. \& Fuller-Tyszkiewicz, M. (2016). Body Image Concerns as Predictors of Psychology Students' Confidence with Clients. Journal of Applied Biobehavioral Research, 21(4), 253-261.

10. De la Serna, I. (2004). Introducción: alteraciones de la imagen corporal. Monografías de Psiquiatría, 16(2), 1-2.

11. Hernández, R., Fernández, C. \& Baptista, P. (2014). Metodología de la investigación. México: McGraw- Hill.

12. Medina-Gómez, M. B., Martínez-Martín, M. A., Escolar-Llamazares, M. C., González-Alonso, Y. \& Mercado-Val, E. (2019). Anxiety and body dissatisfaction in university students. Acta Colombiana de Psicología, 22(1), 13-21. doi: http://www.dx.doi.org/10.14718/ ACP.2019.22.1.2

13. Ornelas, M., Gastélum, G., Blanco, H. \& Peinado, J. E. (2014). Análisis psicométrico de la prueba Body Image Anxiety Scale versión rasgo en alumnos de educación superior. Perfiles Educativos, 36(144), 120-137.

14. Reed, D. L., Thompson, K., Brannick, M. T. \& Sacco, W. P. (1991). Development and Validation of the Physical Appearance State and Trait Anxiety Scale (PASTAS. Joumal of Anxiety Disorders, 5(4), 323-332.

15. Robles, G. S. I., Molina, R. G., Solís, O. B., Zueck, M. D. C., Blanco, H., González, M. A., . . Jiménez, C. (2020). Perceived Body Image in Psychology Students from Yucatán, Mexico. European Scientific Journal, 16(14), 27-37. doi: https://doi.org/10.19044/esj.2020.v16n14p27

16. Rosen, J. C. (1995). The nature of body dysmorphic disorder and treatment with cognitive-behavior therapy. Cognitive and Behavioral Practice, 2, 143-166.

17. Salaberria, K., Rodríguez, S. \& Cruz, S. (2007). Percepción de la imagen corpora. Osasunaz, 8(2), 171-183.

18. Schilder, P. (1935). Image and appearance of the human body. Londre: Kegan Paul, Trench Trubner and Co.

19. Sepúlveda, A. R., Gandarillas, A. \& Carrobes, J. A. (2004). Prevalencia de trastornos del comportamiento alimentario en la 
población universitaria. Trabajo presentado en $5^{\circ}$ Congreso Virtual de Psiquiatría.

https://psiquiatria.com/trastornos_de_alimentacion/prevalencia-detrastornos-del-comportamiento-alimentario-en-la-poblacionuniversitaria/

20. Shamsuddin, K., Fadzil, F., Ismail, W. S. W., Shah, S. A., Omar, K., Muhammad, N. A., . . . Mahadevan, R. (2013). Correlates of depression, anxiety and stress among Malaysian university students. Asian Journal of Psychiatry, 6(4), 318-323. doi: https://doi.org/10.1016/j.ajp.2013.01.014

21. Sierra, J. C., Ortega, V. \& Zubeidat, I. (2003). Ansiedad, angustia y estrés: tres conceptos a diferenciar. Revista mal-estar e subjetividade, $3(1), 10-59$.

22. Vaquero-Cristóbal, R., Alacid, F., Muyor, J. M. \& López-Miñarro, P. A. (2013). Body image: literature review. Nutrición Hospitalaria, 28(1), 27-35. doi: http://dx.doi.org/10.3305/ nh.2013.28.1.6016.

23. Zueck, M. C., Blanco, L. H., Ornelas, M. \& Gastélum, G. (2015). Invarianza Factorial de la Escala de Ansiedad Asociada a la Imagen Corporal en Universitarios Hombres y Mujeres. Formación Universitaria, 8(5), 91-96. doi: 10.4067/S0718-50062015000500010. 